\section{Keratocyte apoptosis and corneal antioxidant enzyme activities after refractive corneal} surgery

K Bilgihan ${ }^{1}$, A Bilgihan ${ }^{2}, \cup$ Adiguzel ${ }^{1}$, C Sezer $^{3}$, $\mathrm{O}$ Yis $^{2}, \mathrm{G} \mathrm{Akyol}{ }^{3}$ and B Hasanreisoglu ${ }^{1}$

\begin{abstract}
Purpose Refractive corneal surgery induces keratocyte apoptosis and generates reactive oxygen radicals (ROS) in the cornea. The purpose of the present study is to evaluate the correlation between keratocyte apoptosis and corneal antioxidant enzyme activities after different refractive surgical procedures in rabbits.
\end{abstract}

Methods Rabbits were divided into six groups. All groups were compared with the control group (Group 1), after epithelial scraping (Group 2), epithelial scrape and photorefractive keratectomy (PRK) (traditional PRK: Group 3), transepithelial PRK (Group 4), creation of a corneal flap with microkeratome (Group 5) and laserassisted in situ keratomileusis (LASIK, Group 6). Terminal deoxyribonucleotidyl transferase-mediated dUTP-digoxigenin nickend labelling assay (to detect DNA fragmentation in situ) and light microscopy were used to detect apoptosis in rabbit eyes. Glutathione peroxidase (Gpx) and superoxide dismutase (SOD) activities of the corneal tissues were measured with spectrophotometric methods.

Results Corneal Gpx and SOD activities decreased significantly in all groups when compared with the control group $(P<0.05)$ and groups 2, 3 and 6 showed a significantly higher amount of keratocyte apoptosis $(P<0.05)$. Not only a negative correlation was observed between corneal SOD activity and keratocyte apoptosis (cc: -0.3648 ) but Gpx activity also showed negative correlation with keratocyte apoptosis (cc: -0.3587 ). Conclusion The present study illustrates the negative correlation between keratocyte apoptosis and corneal antioxidant enzyme activities. This finding suggests that ROS may be partly responsible for keratocyte apoptosis after refractive surgery. Eye (2002) 16, 63-68. DOI: 10.1038/ sj/EYE/6700065

Keywords: keratocyte apoptosis; SOD; Gpx; refractive surgery

Apoptosis is a form of cellular suicide which describes the process of leaves falling from trees or petals from flowers. The significance of apoptosis is based on the fact that apoptotic cells tend to be 'environmentally friendly' and package their contents into membrane-bound vesicles, ready for ingestion by phagocytic cells without releasing their contents into the intracellular matrix. Hence, there is no inflammatory response. ${ }^{1}$ Keratocyte apoptosis has been demonstrated after refractive surgery in previous studies. ${ }^{2-5}$ It is believed that clinical differences in regression and haze in patients who have been treated with photorefractive keratectomy (PRK) and/or laser in situ keratomileusis (LASIK) are attributable to diminished keratocyte apoptosis and an attenuated wound healing response in LASIK treatment. ${ }^{2,4}$ In addition, keratocyte apoptosis may be induced posterior corneal ectasia after LASIK surgery.

On the other hand, excimer laser tissue interaction induces polymorphonuclear (PMN) cell infiltration and free radical formation in the cornea. ${ }^{6-10}$ The harmful effects of reactive oxygen species (ROS) are widely known, and the pharmacological treatments to reduce oxidative damage in corneal tissue after PRK is under investigation. ${ }^{11,12}$ Superoxide dismutase (SOD) and glutathione peroxidase
'Department of Ophthalmology Gazi University Medical School

Ankara, Turkey

${ }^{2}$ Department of Biochemistry Gazi University Medical School

Ankara, Turkey

${ }^{3}$ Department of Pathology Gazi University Medical School

Ankara, Turkey

Correspondence: $\mathrm{K}$ Bilgihan

Umit mah.30.sok. Defne Sitesi 3.Blok 53/10 06530 Umitkoy Ankara, Turkey Fax: 903122125794 E-mail: bilgihan@ med.gazi.edu.tr 
(Gpx) are the main antioxidant enzymes which protect the cornea from radical injury. ${ }^{13,14}$ Catalase which has little activity in the cornea is another antioxidant enzyme of it. ${ }^{15}$

Recent studies have shown the relation between ROS and apoptosis. Apoptosis and necrosis induced by ROS have been demonstrated in bovine corneal endothelial cells. ${ }^{10}$ In another study, ROS were found partly responsible for keratocyte apoptosis after excimer laser photoablation. ${ }^{9}$

In the present study, the correlation between keratocyte apoptosis and corneal antioxidant enzyme activities (SOD and Gpx) was evaluated after different refractive surgical procedures in rabbits.

\section{Material and methods}

Twenty one New Zealand white rabbits (average weight $2.5-3.4 \mathrm{~kg}$ ) were used in the study. Animals were divided into six groups and each group consisted of seven eyes. Anaesthesia was induced by an intramuscular injection of $25 \mathrm{mg} / \mathrm{kg}$ ketamin $\mathrm{HCl}, 2.5$ $\mathrm{mg} / \mathrm{kg}$ xylazine and topical proparacaine hydrochloride.

Group 1: Consisted of unwounded eyes and served as the control group.

Group 2: The corneal epithelium was removed by a blunt spatula (Visitec, Sarasota, USA) (epithelial scrape). Group 3: Corneal photoablation (59 $\mu \mathrm{m},-5$ diopter) was performed after epithelial removal (traditional PRK).

Group 4: The corneal epithelium was removed by using excimer laser in PTK mode, then stromal photoablation was performed ( $59 \mu \mathrm{m},-5$ diopter) (transepithelial PRK).

Group 5: A hinged corneal flap of approximately $150 \mu \mathrm{m}$ thickness and $7.0 \mathrm{~mm}$ in diameter was created by using an automated corneal microkeratome (Eyetech Vision, La Jolla, CA, USA), then the flap was repositioned without any laser treatment (corneal flap). Group 6: A hinged corneal flap was created and laser ablation ( $63 \mu \mathrm{m},-5$ diopter) was performed in the stromal bed in group 6 (LASIK).

The eyes were irradiated with 193-nm excimer laser (MEL 60, Aesculap-Meditec, Jena, Germany) under in vivo conditions. The fluency at the cornea was 220 $\mathrm{mJ} / \mathrm{cm}^{2}$, firing rate of $20 \mathrm{~Hz}$, and diameter of ablation zone was $6 \mathrm{~mm}$ in all groups.

Four hours after the corneal surgery, the animals were euthanized with $100 \mathrm{mg} / \mathrm{kg}$ intravenous pentobarbital injection because the previous studies illustrated that the maximum staining of the apoptotic keratocytes with TUNEL assay was 4 hours after the corneal surgery. ${ }^{4}$ Institutional guidelines of ARVO regarding animal experimentation were followed in the study.

\section{Histological study}

Central corneal tissues were removed by using a 7.5$\mathrm{mm}$ trephine and divided into two pieces, one half of the tissues were used for enzymatic investigation and the other half of the cornea was fixed in $10 \%$ formaldehyde solution for two hours and then embedded vertically in paraffin. Paraffin tissue blocks were cut as 4 micron thickness, following routine deparaffinisation, proteinase $\mathrm{K}$ (Applipere, Oncor 130202) being applied for 15 minutes. The cornea was stained according to the manufacturer's instructions with terminal deoxyribonucleotidyl transferasemediated dUTP-digoxigenin nick-end labelling (TUNEL) assay by using ApopTag plus peroxidase in situ apoptosis detection kit (S-7101, Oncor).

Counterstaining of the specimen was made in $0.5 \%$ methyl green. Rat breast tissue was used as a positive control material (Figure 1). Four sections from each specimen were evaluated in the light microscopy at higher magnification fields $(\times 400)$ and TUNEL + keratocytes were counted by the same observer. The number of stained keratocytes were counted in 40 different fields for each eye and the mean values were calculated for each group.

\section{Enzyme assay}

Each corneal tissue was homogenised in $1 \mathrm{ml}$ of $0.1 \mathrm{M}$ Tris/ $\mathrm{HCl}$ buffer, $\mathrm{pH}$ 7.2, containing $154 \mathrm{mM} \mathrm{NaCl}$. Homogenate was centrifuged at $13000 \mathrm{rpm}$ for $15 \mathrm{~min}$.

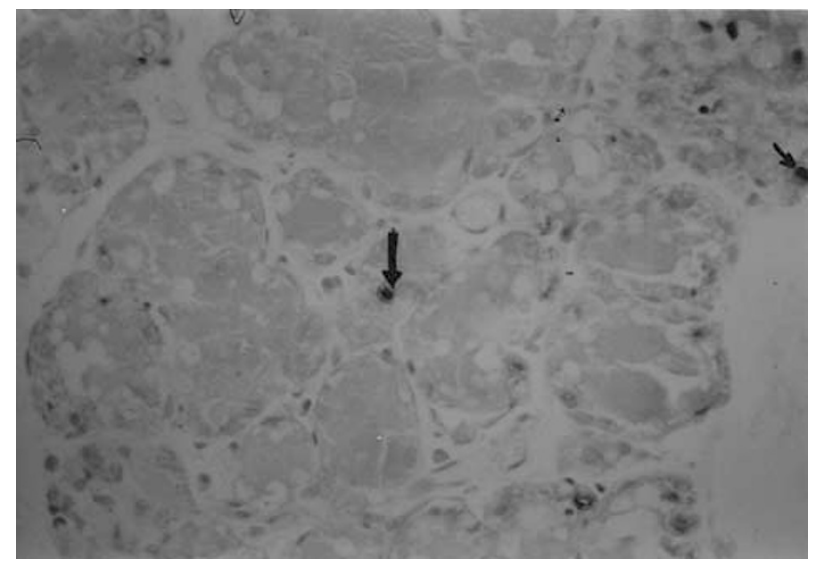

Figure 1 Control slide, normal female rodent mammary gland. Cells positively stained with apopTag are shown (arrows). In situ hybridisation $400 \times$, 3,3'-diaminobenzidine (DAB). 
The protein concentration of the supernatant was measured by the method of Lowry et al ${ }_{1}^{16}$ glutathione peroxidase activity was measured by using a modification of the coupled assay procedure of Paglia and Valentine ${ }^{17}$ and the results were expressed as nmoles NADPH oxidised per minute per mg protein. SOD activities of the samples were determined by inhibition of nitroblue tetrazolium reduction with xanthine-xanthine oxidase which is used as a superoxide generator in the same supernatants. ${ }^{18}$ The results were expressed as units/mg protein. One unit of SOD is defined as the amount of protein that inhibits the rate of nitroblue tetrazolium reduction by $50 \%$.

\section{Statistical analyses}

The ANOVA test was used for the evaluation of the corneal antioxidant enzyme activities and keratocyte apoptosis. If $P$ values less than 0.05 were found, statistical significance was mentioned.

Finally, a partial correlation coefficient test was used for the evaluation of the correlation between keratocyte apoptosis and antioxidant enzyme activities.

Data were analysed using SPSS 9.0 for Windows (SPSS Inc).

\section{Results}

The number of keratocytes that become stained in the TUNEL assay after different refractive surgical procedures is given in Figure 2. Groups 2, 3 and 6 showed a significantly higher amount of keratocyte apoptosis when compared with the control group $(P<0.05)$. TUNEL positive keratocytes were observed in the anterior stroma in groups 2, 3 and 4 (Figures 3 and 4). However, in the LASIK and flap groups, positively stained cells were observed surrounding the lamellar incision.

Corneal SOD activities decreased significantly in all groups when compared with the control group $(P<0.05)$, the lowest corneal SOD activity was observed after traditional PRK, and the highest corneal SOD activity was observed after LASIK (Figure 5).

Gpx activities of the groups are given in Figure 6. Corneal Gpx activities were found significantly decreased in all groups when compared with the control group $(P<0.05)$ the lowest corneal Gpx activities being observed in traditional PRK and LASIK groups.

The correlation between keratocyte apoptosis and corneal SOD activity is given in Figure 7. Negative correlation was observed between these two groups (correlation coefficient: -0.3648 ). The correlation

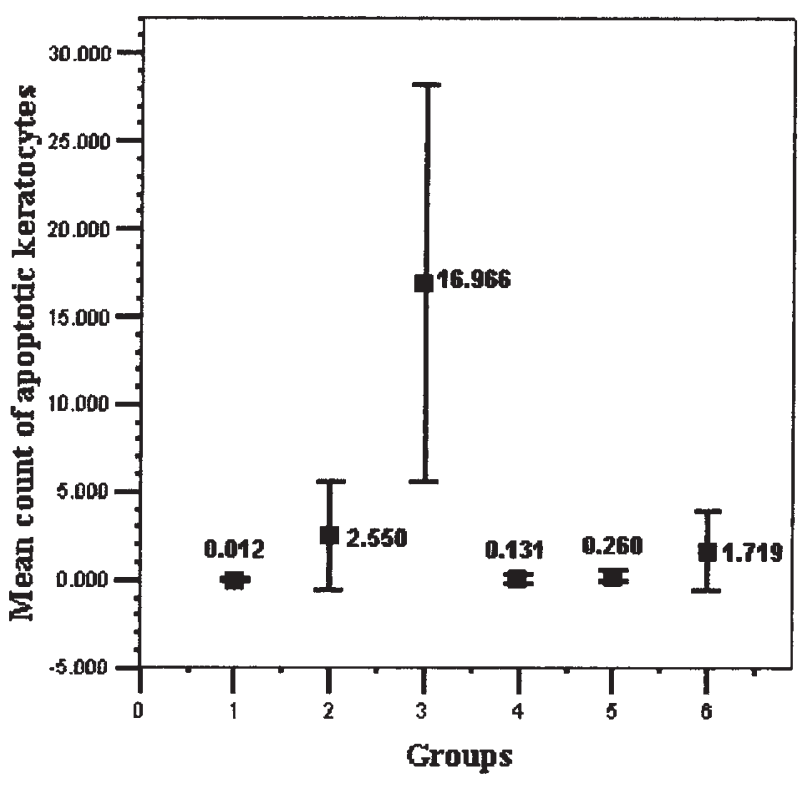

Figure 2 Mean count of apoptotic keratocytes after different corneal surgical manipulations are seen. Labels represent means, and error bars represent standard deviations. Group 1: Control. Group 2: Epithelial scrape $(P<0.05)$. Group 3: Traditional PRK $(P<0.05)$. Group 4: Transepithelial PRK $(P>0.05)$. Group 5: Corneal flap: $(P>0.05)$. Group 6: LASIK $(P<0.05)$.

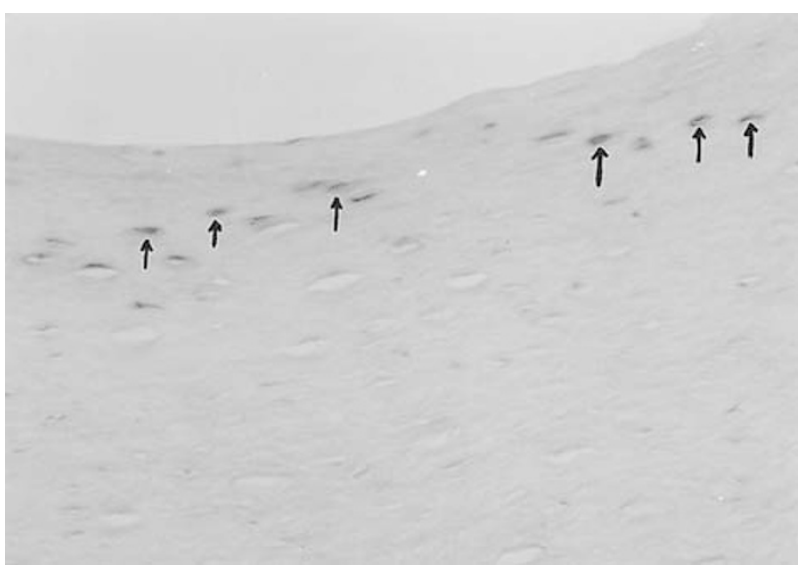

Figure 3 Apoptotic keratocytes (arrow heads) in the anterior corneal stroma were stained with apopTag, in the traditional PRK group. In situ hybridisation $400 \times$, DAB.

between keratocyte apoptosis and corneal Gpx activity is given in Figure 8; corneal Gpx activity also showed negative correlation with keratocyte apoptosis (correlation coefficient: -0.3587 ).

\section{Discussion}

Apoptosis is a complex controlled and altruistic way of programmed cell death in which damaged or injured cells commit suicide to allow the neighbouring cells to continue to proliferate without being affected by the 


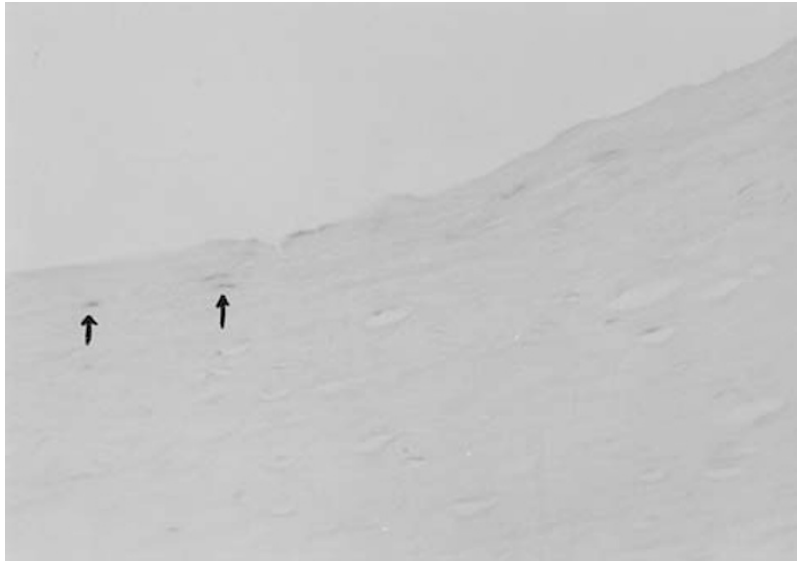

Figure 4 Low intensive staining (arrow head) is noticed in the anterior corneal stroma in the transepithelial PRK group. In situ hybridisation $400 \times$, DAB.

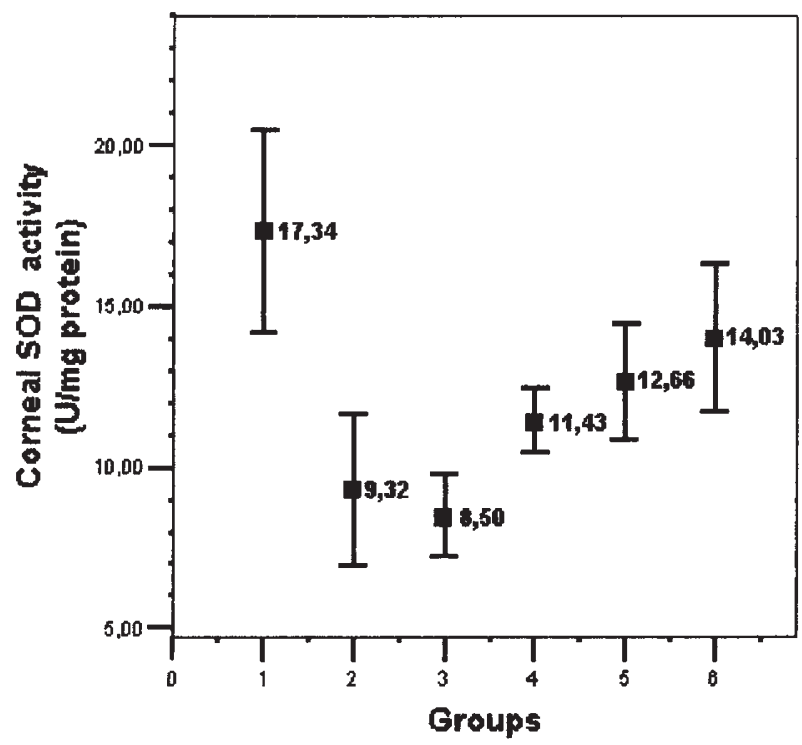

Figure 5 Corneal SOD activities after different refractive surgical manipulations. Labels represent means, and error bars represent standard deviations. Group descriptions as in figure caption 2.

death of the neighbour. ${ }^{1}$ Apoptotic cells participate in their own death, which is characterised by nuclear condensation and fragmentation with intact cytoplasmic organales.

Previous studies have shown that keratocyte apoptosis is likely mediated by cytokines such as Fas ligand and interleukin-1 after PRK and LASIK. ${ }^{4}$ In a recent study the release of soluble Fas and Fas ligand was found significantly increased after PRK in human tears. ${ }^{19}$ The apoptotic keratocytes are replenished within a few days through proliferation and migration of remaining keratocytes, but the new keratocytes are activated and thought to produce more collagen,

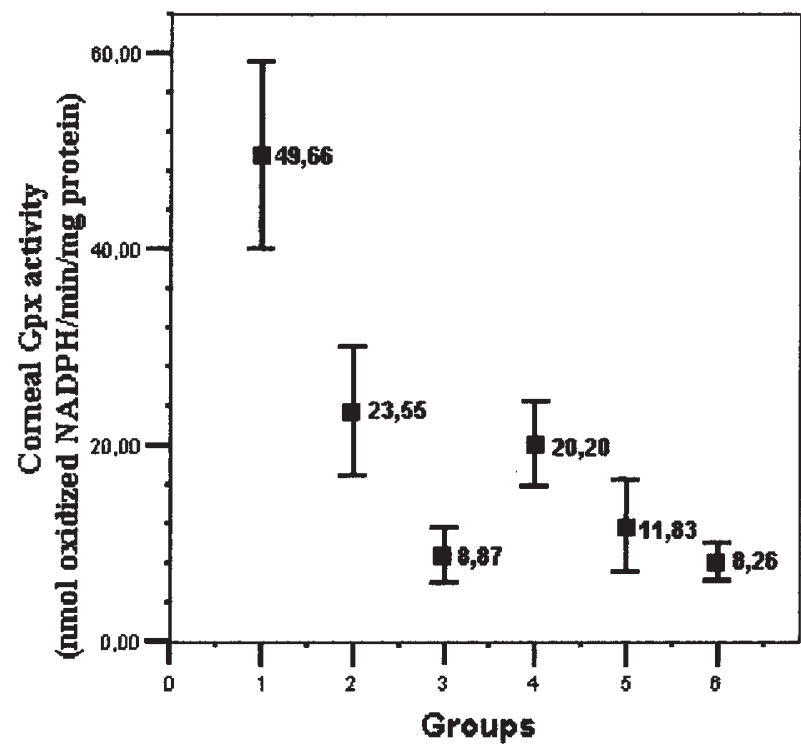

Figure 6 Corneal Gpx activities after different refractive surgical manipulations. Labels represent means, and error bars represent standard deviations. Group descriptions as in figure caption 2 .

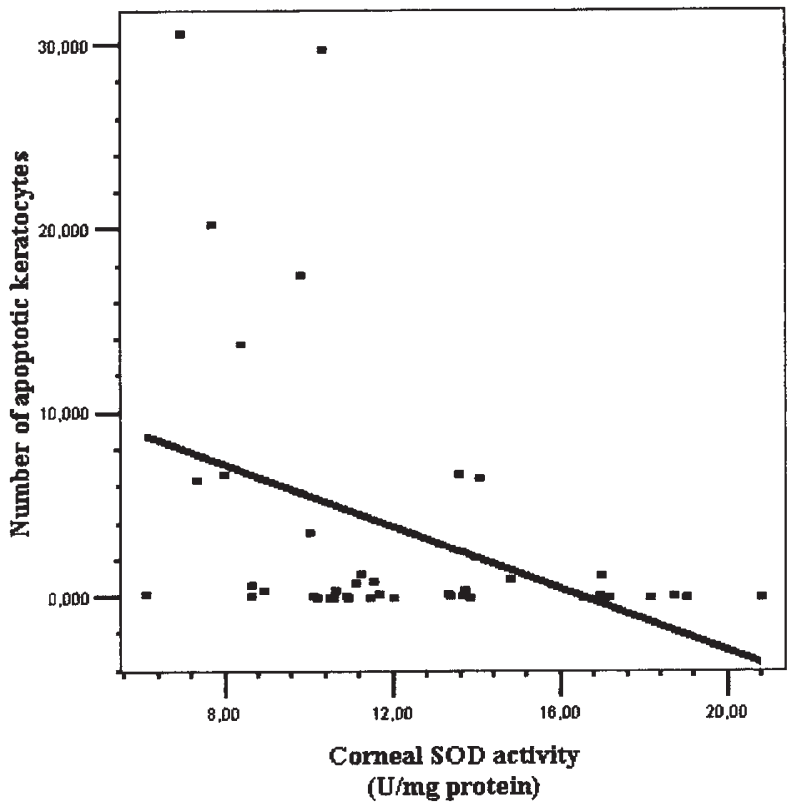

Figure 7 The correlation between keratocyte apoptosis and corneal SOD activity (correlation coefficient $=0.4034, P<0.05$ ).

glycosaminoglycans, growth factors and other wound healing components., ${ }^{4,5}$

The other harmful effect of excimer laser surgery is induction of ROS. UV radiation, corneal PMN cell infiltration and thermal increase are the probable sources of ROS after PRK. ROS induce the peroxidation of fatty acids or lipids and proteins of the cell membranes, degrade corneal stromal tissue by 


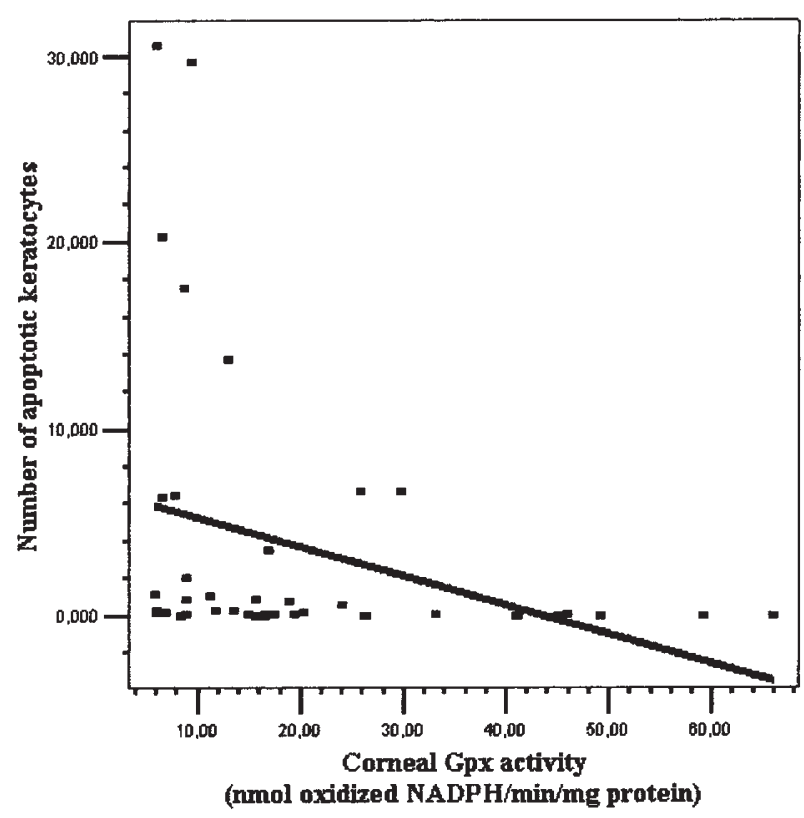

Figure 8 The correlation between keratocyte apoptosis and corneal Gpx activity (correlation coefficient $=0.5840, P<0.01$ ).

scission of covalent bonds or enhancing their susceptibility to hydrolytic enzymes. ${ }^{7,11}$ Previous studies have demonstrated that excimer laser keratectomy increases the corneal temperature, decreases the superoxide dismutase activity of the aqueous humour, and induces lipid peroxidation in the superficial corneal stroma. ${ }^{7,8}$ Excimer laser treatment also changes the corneal activities of aldehyde dehydrogenase and glutathione S-transferase, these two enzymes play an important role in detoxification of aldehydes, which are generated from free radical reactions..$^{20}$ Excimer laser has induced radical formation which has also been demonstrated by electron spinresonance recently. ${ }^{9}$

The cornea protects itself from radical injury by antioxidant enzymes, and free radical scavengers, such as vitamin $\mathrm{E}$ and vitamin $\mathrm{C}$. The protective effect of vitamin $C$ from lipid peroxidation after PRK has been demonstrated previously. ${ }^{11}$ The inhibitory effect of topical vitamin $\mathrm{E}$ on excessive corneal wound healing after PRK has been shown. ${ }^{12}$

The relation between ROS and apoptosis has been demonstrated..$^{9,10}$ ROS may lead to apoptosis when antioxidant capacity of the tissue is suppressed and recent data confirm that oxygen free radicals can be the mediators of apoptosis. ${ }^{21}$ In addition, antioxidant agents may prevent apoptosis. ${ }^{22}$ The preventive effect of topical vitamin E on keratocyte apoptosis after PRK has been demonstrated and it is suggested that free radicals might be partly responsible for keratocyte apoptosis after excimer laser keratectomy. ${ }^{23}$
Corneal tissue has high SOD and Gpx activity. ${ }^{13,14}$ SOD catalyses the superoxide radical dismutation to hydrogen peroxide and molecular oxygen. ${ }^{15} \mathrm{Gpx}$ constitutes a major defence system against oxidative damage to essential intracellular low molecular weight compounds, proteins and poly-unsaturated fatty acids, particularly as an effective reduction of hydroperoxide to water. ${ }^{6}$

Corneal SOD and Gpx activities have significantly decreased in all groups in the present study $(P<0.05)$. The result confirms that corneal epithelial scraping, stromal photoablation, preparing a corneal flap with microkeratoma and LASIK treatment decrease the corneal antioxidant enzyme activities in the early postoperative period and cornea become more susceptible to the harmful effects of ROS after these manipulations. It is interesting that the highest amount of apoptotic keratocytes have been observed in the traditional PRK group and the lowest enzyme activities have also been observed in the same group. This result suggests that traditional PRK induces more injury and more corneal wound healing response when compared with transepithelial PRK and LASIK.

The other finding is that the lowest amount of apoptotic keratocytes have been observed in the transepithelial PRK group, but the SOD and Gpx activities of this group have significantly decreased $(P<0.05)$. The high activities of SOD and Gpx have been demonstrated in the corneal epithelium. ${ }^{13,14}$ Therefore, removing the epithelial tissue either with the spatula or excimer laser, removes Gpx and SOD from the cornea. It is believed that the harmful effects of ROS might become more prominent after epithelial removal. Although transepithelial PRK has been associated with the lowest amount of keratocyte apoptosis, decreased corneal antioxidant enzyme activities suggest that corneal injury and wound healing response may be higher than LASIK after transepithelial PRK.

The results of the present study strictly suggest that excimer laser tissue interaction reduces the corneal Gpx activity more than SOD activity. A significant decrease in Gpx activity after epithelial scraping has been observed but excimer laser stromal photoablation has induced additional depletion in the Gpx activity of the cornea. Gpx activity was found predominantly in the corneal epithelium and endothelium. UV radiation may also inhibit the endothelial Gpx activity because the lowest Gpx activities have been observed in traditional PRK and LASIK groups.

In conclusion, keratocytes undergo apoptosis after refractive surgery in response to mechanical epithelial removal, and excimer laser stromal ablation. 
Transepithelial PRK induces minimal keratocyte apoptosis that is less than all other refractive surgical procedures but low levels of corneal antioxidant enzyme activities suggest that corneal injury and wound healing response may be higher than LASIK after transepithelial PRK. Corneal epithelial scraping, stromal photoablation, preparing a corneal flap with microkeratoma and LASIK treatment decrease the SOD and Gpx activities of the corneal tissue and cornea becomes more susceptible to the harmful effects of ROS after these manipulations. Transepithelial PRK is probably a safer technique than traditional PRK because the antioxidant enzyme activities of the transepithelial PRK group were found higher. Excimer laser photoablation may probably inhibit endothelial Gpx activity because the lowest Gpx activities have been observed in traditional PRK and LASIK groups. This study demonstrates the negative correlation between keratocyte apoptosis and corneal antioxidant enzyme activities, which suggests that ROS may be partly responsible for keratocyte apoptosis after refractive surgery.

\section{References}

1 Samali A, Gorman AM, Cotter TG. Apoptosis-the story so far... Experientia 1996; 52: 933-941.

2 Wilson SE. Molecular cell biology for the refractive corneal surgeon: programmed cell death and wound healing. J Refract Surg 1997; 13: 171-175.

3 Wilson SE. Everett Kinsey Lecture. Keratocyte apoptosis in refractive surgery CLAO J 1998; 24: 181-185.

4 Helena MC, Baerveldt F, Kim WJ, Wilson SE. Keratocyte apoptosis after corneal surgery. Invest Ophthalmol Vis Sci 1998; 39: 276-283.

5 Krueger RR. Could apoptosis change the way we do PRK? J Refract Surg 1998; 14: 494-496.

6 Landry RJ, Pettit GH, Hahn DW et al. Preliminary evidence of free radical formation during argon fluoride excimer laser irradiation of corneal tissue. Lasers Light Ophthalmol 1994; 6: 87-90.

7 Bilgihan K, Bilgihan A, Akata F, Turkozkan N, Hasanreisoglu B. Excimer laser corneal surgery and free oxygen radicals. Jpn J Ophthalmol 1996; 40: 154-157.

8 Hayashi S, Ishimoto S, Wu G, Wee W, Rao N, McDonnell $\mathrm{P}$. Oxygen free radical damage in the cornea after excimer laser therapy. Br J Ophthalmol 1997; 81: 141-144.
9 Shimmura S, Masumizu T, Nakai Y et al. Excimer laser induced hydroxyl radical formation and keratocyte death in vitro. Invest Ophthalmol Vis Sci 1999; 40: 1245-1249.

10 Cho KS, Lee EH, Choi JS et al. Reactive oxygen species induced apoptosis and necrosis in bovine corneal endothelial cells. Invest Ophthalmol Vis Sci 1999; 40: 911919 .

11 Kasetsuwan N, Wu FM, Hsieh F et al. Effect of topical ascorbic acid on free radical tissue damage and inflammatory cell influx in the cornea after excimer laser corneal surgery. Arch Ophthalmol 1999; 117: 649-562.

12 Bilgihan K, Ozdek S, Ozogul C, Gurelik G, Bilgihan A, Hasanreisoglu B. Topical vitamin $\mathrm{E}$ and hydrocortisone acetate treatment after photorefractive keratectomy. Eye 2000; 14: 231-237.

13 Behnding A, Svensson B, Marklund SL, Karlsson K. Superoxide dismutase isoenzymes in the human eye. Invest Ophthalmol Vis Sci 1998; 39: 471-475.

14 Atalla LR, Sevanian A, Rao NA. Immunohistochemical localisation of glutathione peroxidase in ocular tissue. Cur Eye Res 1988; 7: 1023-1027.

15 Mayer U. Comparative investigations of catalase activity in different ocular tissues of cattle and man. Albrecht Von Graefes Arch Klin Exp Ophthalmol 1980; 213: 261-265.

16 Lowry OH, Rosebrough NJ, Farr AJ, Randall RJ. Protein measurement with folin phenol reagent. J Biol Chem 1951; 193: 265-266.

17 Paglia DE, Valentine WN. Studies on the quantitative and qualitative characterization of erythrocyte glutathione peroxidase. J Lab Clin Med 1967; 70: 158-169.

18 Sun Y, Oberley LW, Li Y. A simple method for clinical assay of superoxide dismutase. Clin Chem 1988; 34: 497500

19 Tuominen I, Vesaluoma M, Teppo AM, Gronhagen-Riska C, Tervo T. Soluble Fas and Fas ligand in human tear fluid after photorefractive keratectomy. Br J Ophthalmol 1999; 83: 1360-1363.

20 Bilgihan K, Bilgihan A, Hasanreisoglu B,Turkozkan N. Corneal aldehyde dehydrogenase and glutathione Stransferase activity after excimer laser keratectomy in guinea pigs. Br J Ophthalmol 1998; 82: 300-302.

21 Stoian I, Oros A, Moldoveanu E. Apoptosis and free radicals. Biochem Mol Med 1996; 59: 93-97.

22 Tagami M, Ikeda K, Yamagata K, Nara Y, Fujino H, Kubota A et al. Vitamin E prevents apoptosis in hippocampal neurons caused by cerebral ischemia and reperfusion in stroke-prone spontaneously hypersensitive rats. Lab Invest 1999; 79: 609-615.

23 Bilgihan K, Adiguzel U, Sezer C, Akyol G, Hasanreisoglu B. Effects of topical vitamin E on keratocyte apoptosis after traditional photorefractive keratectomy. Ophthalmologica 2001; 215: 192-196. 